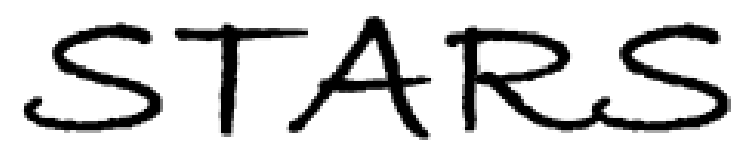

University of Central Florida

STARS

$1-1-1992$

\title{
The Harmonic Lattice, Recoilless Transitions, And The Coherent State
}

David S. Bateman

University of Central Florida

Subir K. Bose

University of Central Florida

Binayak Dutta-Roy

University of Central Florida

Manoranjan Bhattacharyya

Find similar works at: https://stars.library.ucf.edu/facultybib1990

University of Central Florida Libraries http://library.ucf.edu

This Article is brought to you for free and open access by the Faculty Bibliography at STARS. It has been accepted for inclusion in Faculty Bibliography 1990s by an authorized administrator of STARS. For more information, please contact STARS@ucf.edu.

\section{Recommended Citation}

Bateman, David S.; Bose, Subir K.; Dutta-Roy, Binayak; and Bhattacharyya, Manoranjan, "The Harmonic Lattice, Recoilless Transitions, And The Coherent State" (1992). Faculty Bibliography 1990s. 406.

https://stars.library.ucf.edu/facultybib1990/406

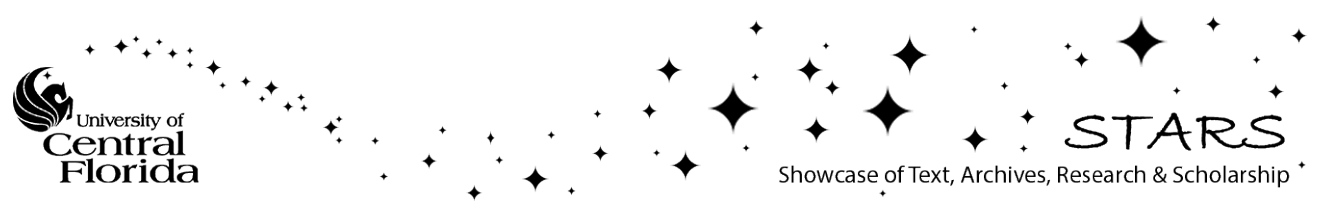


The harmonic lattice, recoilless transitions, and the coherent state

David S. Bateman, Subir K. Bose, Binayak Dutta-Roy, and Manoranjan Bhattacharyya

Citation: American Journal of Physics 60, 829 (1992); doi: 10.1119/1.17064

View online: https://doi.org/10.1119/1.17064

View Table of Contents: https://aapt.scitation.org/toc/ajp/60/9

Published by the American Association of Physics Teachers

\section{ARTICLES YOU MAY BE INTERESTED IN}

Regularization and renormalization in scattering from Dirac delta potentials American Journal of Physics 66, 1101 (1998); https://doi.org/10.1119/1.19051

The mapping of the Coulomb problem into the oscillator ${ }^{a)}$

American Journal of Physics 60, 833 (1992); https://doi.org/10.1119/1.17065

The Born-Oppenheimer approximation: A toy version

American Journal of Physics 72, 389 (2004); https://doi.org/10.1119/1.1625927

How metals bind: The deformable-jellium model with correlated electrons

American Journal of Physics 71, 1048 (2003); https://doi.org/10.1119/1.1590653

Renormalization of a model quantum field theory

American Journal of Physics 60, 1013 (1992); https://doi.org/10.1119/1.16980

The Mössbauer effect explained

American Journal of Physics 66, 593 (1998); https://doi.org/10.1119/1.18911

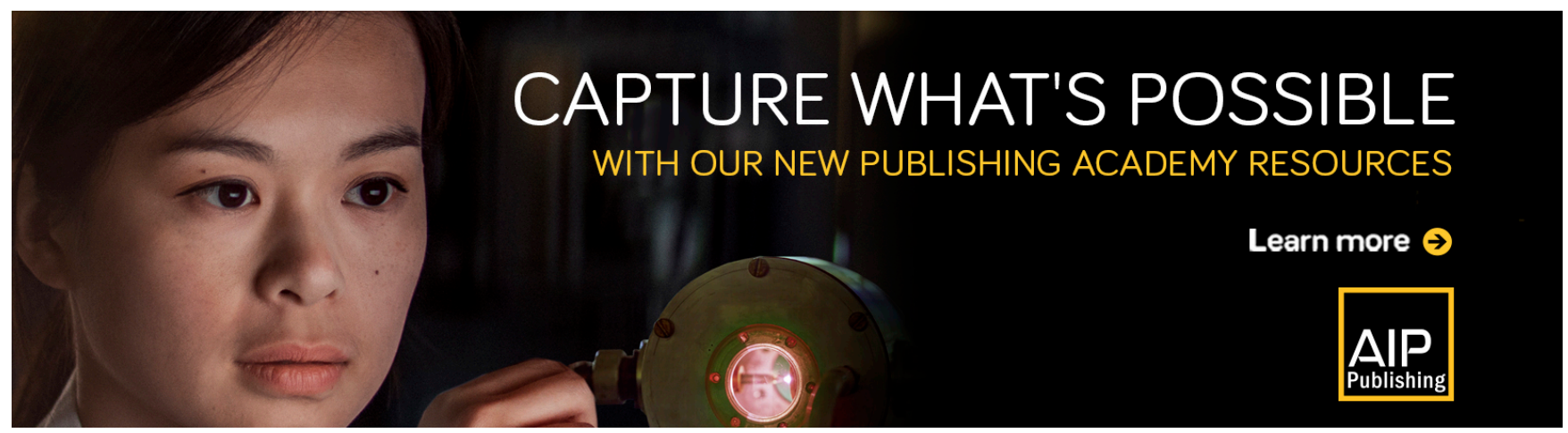




\author{
David S. Bateman, Subir K. Bose, and Binayak Dutta-Roya) \\ Department of Physics, University of Central Florida, Orlando, Florida 32816 \\ Manoranjan Bhattacharyya \\ Saha Institute of Nuclear Physics, Calcutta, India
}

(Received 12 December 1990; accepted 28 October 1991)

The probability for recoilless transitions, relevant for the understanding of $x$-ray scattering from atoms bound in a crystal (applicable also to elastic scattering of neutrons from solids and to the Mössbauer effect), given by the Debye-Waller factor, is derived in a novel manner using the coherent state basis for the normal mode oscillators describing the harmonic lattice, a method which, while being simple and elegant, also reveals the relationship to a heuristic classical discussion of the problem.

\section{THE PROBABILITY FOR RECOILLESS TRANSITIONS}

The intensity distribution in $\mathrm{x}$-ray diffraction spots from atoms in a lattice (made diffuse by thermal vibrations) is given by the Debye-Waller factor. ${ }^{1}$ The result also applies to the determination of the fraction of gamma rays emitted (or absorbed) resonantly (without change in energy due to recoil) by a nucleus bound in a crystal that occurs in the celebrated Mössbauer effect as well as for the case of elastic scattering of neutrons from solids. The probability that a nucleus in the lattice emits (or absorbs) a photon of momentum $p$ without any change in the state of the lattice is given by the square modulus of the overlap between the state vector $e^{i \mathrm{p} \cdot \mathrm{X} / \hbar}\left|L_{i}\right\rangle$, corresponding to the lattice with a "site," displaced by $\mathbf{X}$ with respect to its equilibrium position and recoiling with momentum $p$, and the state $\left|L_{i}\right\rangle$ representing the undisturbed lattice. Of course, with a solid at temperature $T$ it is also essential to include the thermal average (shown below through a second set of angular brackets) thus yielding the basic formula

$$
f=\left|\left\langle\left\langle L_{i}\left|e^{i \mathrm{p} \cdot \mathrm{X} / \hbar}\right| L_{i}\right\rangle\right\rangle_{T}\right|^{2},
$$

from which the Debye-Waller factor $f$ is to be calculated. Here, it needs to be noted that $p$ the photon momentum is a number while $X$ is an operator. Lattice vibrations in a solid, in the harmonic approximation, are described in terms of the normal modes, with corresponding coordinates $\left\{\xi_{s}\right\}$, and hence by an ensemble of oscillators with frequencies $\left\{\omega_{s}\right\}$. The state of the lattice can then be specified by providing the oscillator quantum numbers (occupancies) of the different modes, viz., $\left|\left\{n_{s}\right\}\right\rangle$. We can now express the component of $\mathbf{X}$ in the direction of recoil, $\hat{p} \cdot \mathbf{X}$ (where $\hat{p}$ is the unit vector along $\mathbf{p}$ ), in terms of the normal-mode coordinates $\xi_{s}$ for the lattice:

$$
\hat{p} \cdot \mathbf{X}=\sum_{s=1}^{3 N} c_{s} \xi_{s}
$$

with the normalization condition

$$
\sum_{s=1}^{3 N} c_{s}^{2}=1
$$

on the expansion coefficients, where $3 N$ is the number of degrees of freedom for $N$ atoms in three dimensions. Expressing the displacement of the nucleus in terms of the normal modes of the lattice (made possible by the fact that the normal modes are but combinations of the displacements of the nuclei) enables us to discuss the response of the lattice as a whole to the decay or scattering event. The underlying theory and the associated calculation to obtain the probability for such recoilless processes were performed by $\mathrm{Lamb}^{2}$ for neutron scattering from nuclei of atoms bound in a solid, and the corresponding situation for photons has been considered by Mössbauer ${ }^{3}$ and by Visscher. ${ }^{4}$ A simplified derivation for the Mössbauer effect was presented by Lipkin ${ }^{5}$ and also by Weisskopf. ${ }^{6}$ Singwi and Sjölander ${ }^{7}$ used an approach developed by van $\mathrm{Hove}^{8}$ to arrive at the result. Interestingly, a discussion of this problem based on a classical picture for the process provided by Shapiro has also yielded an identical formula, a fact that has been remarked upon as being "unexpected" in the book on Mössbauer effect by Frauenfelder. ${ }^{9}$ The quantal calculations of $f$, referred to above, employ the Fock space (occupation number) representation and involve manipulations of Hermite polynomials and Bessel functions. Furthermore, some of the papers arrive at the exact result, despite certain approximations, which were later shown ${ }^{10}$ to be unnecessary on the basis of mathematical identities involving Hermite polynomials. The present derivation, using the device of coherent states, will avoid these unnecessary complications (using, as we shall see, nothing more complicated than Gaussian integrals) and the "mystery" of the unexpected agreement with the classical result will be clarified.

\section{THE COHERENT STATE}

The energy eigenstates of the proto-typical onedimensional harmonic oscillator, described by the Hamiltonian

$$
H=p^{2} / 2 m+\frac{1}{2} m \omega^{2} x^{2},
$$

are best developed through the introduction of the annihilation and creation operators:

$$
\begin{aligned}
& a \equiv(p-i m \omega x) / \sqrt{2 m \omega \hbar,} \\
& a^{\dagger}=(p+i m \omega x) / \sqrt{2 m \omega \hbar},
\end{aligned}
$$

whereby the normalized $n$th excited state (or the state of $n$ quanta), $|n\rangle$, may be generated from the ground (or 'vacuum') state $|0\rangle$ through the repeated application of the creation operator:

$$
|n\rangle=\left(a^{\dagger}\right)^{n} / \sqrt{n !}|0\rangle
$$


A set of nonstationary normalized states, known as coherent states, ${ }^{11}$ first introduced by Schrödinger, ${ }^{12}$ is obtained through a superposition of the occupation number states, thus

$$
|\alpha\rangle=e^{-|\alpha|^{2} / 2} \sum_{n=0}^{\infty} \frac{\alpha^{n}}{\sqrt{n !}}|n\rangle,
$$

where $\alpha$ is a complex number. These are minimum uncertainty wavepackets as the variance of the position and the momentum for an oscillator in such a state obtainable from

$$
\begin{aligned}
& (\Delta x)^{2}=\left\langle\alpha\left|x^{2}\right| \alpha\right\rangle-\langle\alpha|x| \alpha\rangle^{2}=\hbar / 2 m \omega \equiv l^{2}, \\
& (\Delta p)^{2}=\left\langle\alpha\left|p^{2}\right| \alpha\right\rangle-\langle\alpha|p| \alpha\rangle^{2}=\hbar m \omega / 2=\hbar^{2} / 4 l^{2},
\end{aligned}
$$

satisfy $\Delta x \Delta p=\hbar / 2$. These states have been widely applied to nonlinear optics and laser physics. ${ }^{13-15}$ Their domain of usefulness has been extended to the description of the superfluid state, ${ }^{16}$ pion production, ${ }^{17}$ nuclear structure, ${ }^{18-20}$ infrared problems in quantum electrodynamics, ${ }^{21}$ quantum theory of noise, ${ }^{22}$ plasma physics, ${ }^{23}$ elucidation of classical correspondences, ${ }^{24,25}$ and to various other dreas of physics. $^{26}$

These coherent states, which are in fact eigenstates of the annihilation operator $a$, belonging to the eigenvalue $\alpha$, form a complete (actually an overcomplete) set:

$$
\int|\alpha\rangle\langle\alpha| \frac{d^{2} \alpha}{\pi}=\mathbf{1},
$$

where the integration is over the plane of complex $\alpha$. This "decomposition of the identity" is very useful, in that various operators, and in particular the density operator ${ }^{27} \rho$ (which we shall need when performing thermal averages) can be conveniently incorporated into the formalism, through the so-called $P$ representation: ${ }^{13,14}$

$$
\rho=\int \frac{d^{2} \alpha}{\pi} P(\alpha)|\alpha\rangle\langle\alpha|,
$$

which is but the density operator expressed in the basis of the coherent states. For oscillators in thermal equilibrium at a temperature $T$

$$
P(\alpha)=(1 /\langle n\rangle) e^{-|\alpha|^{2} /\langle n\rangle},
$$

where the average occupancy $\langle n\rangle$ is given by

$$
\langle n\rangle=e^{-\hbar \omega / k T} /\left(1-e^{-\hbar \omega / k T}\right),
$$

$k$ being the Boltzmann constant. These expressions for $P$ are derived in both Refs. 13 and 14. The thermal average of any quantity evaluated in the coherent state basis is thus found by using $P(\alpha)$ as a weight function in determining the mean.

Another great advantage of the coherent state representation resides in the ease with which classical limits can be realized. Thus taking cognizance of the nonstationary character of the state, and putting in the time dependence appropriate for each stationary component $(|n\rangle)$, we have, but for an irrelevant overall phase factor:

$$
|\alpha\rangle_{t}=|\alpha(t)\rangle=\left|\alpha e^{-i \omega t}\right\rangle,
$$

where the notation used above expresses the fact that with the elapse of time the coherent state retains its form, though the eigenvalue $\alpha$ of the annihilation operator $a$, which labels the state will have changed to $\alpha e^{-i \omega t}$. Now writing $\alpha=-i|\alpha| e^{-i \theta}$, it can be verified that

$$
{ }_{t}\langle\alpha|x| \alpha\rangle_{t}=2|\alpha| \sqrt{\hbar / 2 m \omega} \cos (\omega t+\theta),
$$

wherein the limit

$$
\alpha \rightarrow \infty \text { and } \hbar \rightarrow 0: 2|\alpha| \sqrt{\hbar / 2 m \omega} \rightarrow A,
$$

yields, in an explicit manner, the classical correspondence for the time-dependent displacement for an oscillator, viz., $A \cos (\omega t+\theta)$, and moreover the quantal fluctuations expressed through the uncertainties [refer Eqs. (6a) and (6b)] go to zero in this limit.

\section{CALCULATION OF $f$ IN THE COHERENT STATE BASIS}

In order to evaluate the matrix element occurring in $f$ [see Eq. (1)] it is appropriate to express the exponent therein in terms of the normal mode coordinates $\left\{\xi_{s}\right\}$ [vide Eq. (2a)], and, furthermore, to convert these to the corresponding phonon annihilation and creation operators $a_{s}$ and $a_{s}^{\dagger}$ [using relations analogous to Eqs. (4a) and (4b)]:

$$
\xi_{s}=i l_{s}\left(a_{s}-a_{s}^{\dagger}\right),
$$

where for the sake of brevity we have introduced $l_{s}$ $=\sqrt{\hbar / 2 m \omega_{s}}$, with $\omega_{s}$ the modal angular frequency. The matrix element in question therefore becomes

$$
\left\langle\left\{\alpha_{s}\right\}\left|e^{i \mathbf{p} \cdot \mathbf{X} / \hbar}\right|\left\{\alpha_{s}\right\}\right\rangle=\prod_{s}\left\langle\alpha_{s}\left|e^{-\left(p l_{s} / \hbar\right) c_{s}\left(a_{s}-a_{s}^{\dagger}\right)}\right| \alpha_{s}\right\rangle,
$$

where the lattice state is being described through the multimode coherent state $\left|\left\{\alpha_{s}\right\}\right\rangle \equiv \Pi_{s}\left|\alpha_{s}\right\rangle$. It is at this stage that the immense advantage of the coherent state basis begins to manifest itself; for realizing that the kets are eigenstates of the annihilation operator while the bras of the creation operators, the matrix element can be readily evaluated, provided one can move the as to the right of the $a^{\dagger} s$. This is accomplished through the Baker-Hausdorff formula, which generalizes the formula $e^{x} \cdot e^{y}=e^{x+y}$ to the case when the exponents are operators which do not commute. In the special case where two operators $A$ and $B$ are such that their commutator $[A, B]$, is an ordinary number (viz. not an operator), one has:

$$
e^{A+B}=e^{A} \cdot e^{B} \cdot e^{-(1 / 2)[A, B]} .
$$

Thus the operator on the right-hand side of Eq. (13) may be more conveniently expressed, using the BakerHausdorff formula, as

$e^{-\left(p l_{s} \hbar\right) c_{s}\left(a_{s}-a_{s}^{\dagger}\right)}=e^{\left(p l_{s} / \hbar\right) c_{s} a_{s}^{\dagger}} \cdot e^{-\left(p l_{s} / \hbar\right) c_{s} a_{s} \cdot} e^{-\left(p^{2} t_{s}^{2} / \hbar^{2}\right) c_{s}^{2} / 2}$,

where use has been made of $\left[a, a^{\dagger}\right]=1$. Accordingly,

$\left\langle\alpha_{s}\left|e^{-\left(p l l_{s} \hbar\right) c_{s}\left(a_{s}-a_{s}^{\dagger}\right)}\right| \alpha_{s}\right\rangle=e^{-\left(p^{2} t_{s}^{2} / \hbar^{2}\right) c_{s}^{2} / 2} \cdot e^{\left(p l_{s} / \hbar\right) c_{s}\left(\alpha_{s}^{*}-\alpha_{s}\right)}$,

where we have exploited the fact that the coherent states are eigenstates of the annihilation operators. Implementing Eq. (16) in Eq. (13) we arrive at 


$$
\left\langle\left\{\alpha_{s}\right\}\left|e^{i p \cdot X / \hbar}\right|\left\{\alpha_{s}\right\}\right\rangle=e^{-2 i \Sigma_{s} s_{s}\left(p l_{s} / \hbar\right) \operatorname{Im}\left(\alpha_{s}\right)} \cdot e^{-\Sigma_{s}\left(c_{s}^{2} / 2\right)\left(p l_{s} / \hbar\right)^{2}},
$$

which in turn [using Eq. (6b) for each mode] leads to

$$
\begin{gathered}
\left|\left\langle\left\{\alpha_{s}\right\}\left|e^{i \mathrm{p} \cdot \mathrm{X} / \hbar}\right|\left\{\alpha_{s}\right\}\right\rangle\right|^{2} \\
=e^{-p^{2}\left(\Sigma_{s} c_{s}^{2} \tau_{s}^{2}\right) / \hbar^{2}} \\
=e^{-p^{2}\left(\left\langle\mathbf{X}^{2}\right\rangle-\langle\mathbf{X}\rangle^{2}\right) / \hbar^{2}},
\end{gathered}
$$

a generalization of van Hove's result ${ }^{8,9}$ appropriate to a basis with $\langle\mathbf{X}\rangle \neq 0$. To obtain the thermal-averaged probability we use the $P$ representation of the density matrix, taking the $P$-weighted mean, employing the weighting provided by Eq. (8b), to get

$$
\begin{aligned}
\left\langle\left\langle\left\{\alpha_{s}\right\}\left|e^{i \mathrm{p} \cdot \mathrm{X} / \hbar}\right|\left\{\alpha_{s}\right\}\right\rangle\right\rangle_{T} \\
=e^{-(1 / 2) \Sigma_{s} c_{s}^{2}\left(p l_{s} / \hbar\right)^{2}} \prod_{s} \int \frac{d^{2} \alpha_{s}}{\pi\left(n_{s}\right\rangle} e^{-\left|\alpha_{s}\right|^{2} /\left\langle n_{s}\right\rangle} \\
\quad \times e^{-2 i\left(p l_{s} / \hbar\right) c_{s} \operatorname{Im}\left(\alpha_{s}\right)},
\end{aligned}
$$

with

$$
\left\langle n_{s}\right\rangle=e^{-\hbar \omega_{s} / k T} /\left(1-e^{-\hbar \omega_{s} / k T}\right) .
$$

The Gaussian integrals [over $\operatorname{Re}\left(\alpha_{s}\right)$ and $\operatorname{Im}\left(\alpha_{s}\right)$ ] are readily performed [refer to the Appendix] to yield

$$
\begin{aligned}
\left\langle\left\langle\left\{\alpha_{s}\right\}\left|e^{\hat{\mathrm{p}} \cdot \mathrm{X} / \hbar}\right|\left\{\alpha_{s}\right\}\right\rangle\right\rangle_{T} & =e^{-(1 / 2) \Sigma_{s} \kappa_{s}^{2}\left(p l_{s} / \hbar\right)^{2}\left(1+2\left\langle n_{s}\right)\right)} \\
& =e^{-(R / 2) \Sigma_{s}\left(c_{s}^{2} / \hbar \omega_{s}\right) \operatorname{coth}\left(\hbar \omega_{s} / 2 k T\right)},
\end{aligned}
$$

where we have substituted the recoil energy $R$ for $p^{2} / 2 m$. Now the summation over the phonon modes may be replaced by a frequency integral at the cost of introducing a spectral weight function $g(\omega)$, and consequently the Debye-Waller factor becomes

$$
f=e^{-R / 3 N S[g(\omega) / \hbar \omega] \operatorname{coth}(\hbar \omega / 2 k T) d \omega},
$$

where the expansion coefficients $c_{s}$, being frequency independent, ${ }^{9}$ have been put equal to $1 / 3 N$ in deference to the normalization condition [Eq. (2b)].

In the Einstein model of the solid there is a single frequency $\omega_{E}$ and consequently the normalized frequency distribution being

$$
g(\omega)=3 N \delta\left(\omega-\omega_{E}\right),
$$

the Debye-Waller factor becomes

$$
f=e^{-\left[R / k T_{E}\right] \operatorname{coth}\left(T_{E^{\prime}} / T\right)},
$$

where $T_{E}$, the characteristic temperature of the oscillator, is defined through $\hbar \omega_{E}=k T_{E}$. In the Debye model of the solid, on the other hand,

$$
g(\omega)=\left(9 N / \omega_{D}^{3}\right) \omega^{2} \Theta\left(\omega_{D}-\omega\right),
$$

where $\Theta$ is the Heaviside step function, which is zero for negative values of its argument and unity for positive values. A change of variables, $x=\hbar \omega / k T$, enables us to obtain:

$$
f=\exp \left\{-\frac{3 R}{2 k T_{D}}\left[1+4\left(\frac{T}{T_{D}}\right)^{2} \int_{0}^{T_{D} / T} \frac{x d x}{\left(e^{x}-1\right)}\right]\right\},
$$

wherein the Debye temperature has been introduced via $\hbar \omega_{D}=k T_{D}$. This is the expression for the Debye-Waller factor as it appears in the literature. Thus we have demonstrated how the use of coherent states allows the derivation of these well-known results in a straightforward and graceful manner.

The classical derivation of the fraction of unshifted emission from an oscillating source, originally due to Shapiro, as discussed in Frauenfelder's textbook, depends on the basic notion that radiation from an oscillating source (vibrating with frequency $\omega$ ) suffers Doppler shifts, and the amplitude of the unshifted line (of frequency $\Omega$ ), and that of the Doppler wings (of frequencies $\Omega \pm \omega, \Omega \pm 2 \omega$, etc.), could be obtained from the expansion and collection of corresponding terms from:

$$
\exp \left[-i \Omega t+i \mathbf{K} \cdot \mathbf{X}_{0}+i \mathbf{K} \cdot \mathbf{A} \cos (\omega t+\theta)\right],
$$

which represents the wave emitted (with wave vector $\mathbf{K}$ ) by this source which is oscillating with amplitude $A$ about its mean position $\mathbf{X}_{0}$ with a single frequency $\omega$ (as is the case with the Einstein solid). The amplitude of the unshifted line may be extracted from this expression through an integration with respect to the phase $\theta$ (a process that would integrate out to zero all the Doppler wings). It is interesting to observe that the result of this calculation agrees with that arrived at through the method based on the proper quantum treatment of the problem. That this is expected to be the case is made evident in our approach via the coherent state basis, which is ideally suited to expose just such classical correspondences, when it is recognized that Eq. (16), expressing the relevant probability amplitude, is the quantal analog of Eq. (24). If one were now to insert the time-dependent parametrization of $\alpha_{s}$, as introduced through Eqs. (9) and (10), the exact parallelism of the procedure of averaging over $\mathbf{A}$ and $\theta$ for the classical calculation and the integration over the modulus and phase of the complex variable $\alpha$ in the quantum case provides the underlying reason for the mathematical equivalence between the two approaches, at least where $P(\alpha)$ is a function of $|\alpha|$, which holds when we are concerned with the system in thermal equilibrium.

Thus we have demonstrated the efficacy of the coherent state basis for the calculation of the probability for recoilless transitions in crystal lattices, through the ease and elegance of the derivation, and have also exhibited the inherent advantage in making contact with the classical description. It is suggested that the use of the coherent state basis in problems involving phonons could also prove to be advantageous in other problems of solid-state physics too.

\section{APPENDIX}

The process of thermal averaging when we are working in the coherent state basis involves carrying out integrations in the complex $\alpha$-plane using the weighting corresponding to an equilibrium distribution. Thus, for example, carrying out the integration on the right-hand side of Eq. (19a) may be instructive. Writing $\alpha_{s}=\xi+i \eta$ the relevant integral becomes 


$$
\begin{aligned}
& \int \frac{d^{2} \alpha_{s}}{\pi\left\langle n_{s}\right\rangle} e^{-\left|\alpha_{s}\right|^{2} /\left\langle\alpha_{s}\right\rangle} e^{-2 i\left(p l_{s} / \hbar\right) c_{s} \operatorname{lm}\left(\alpha_{s}\right)} \\
& =\frac{1}{\pi\left\langle n_{s}\right\rangle} \int_{-\infty}^{+\infty} d \xi e^{-\xi^{2} /\left\langle n_{s}\right\rangle} \\
& \quad \times \int_{-\infty}^{+\infty} d \eta e^{-\eta^{2} /\left\langle n_{s}\right\rangle-2 i\left(p l_{s} / \hbar\right) c_{s} \eta} .
\end{aligned}
$$

Completing the square in the quadratic form in $\eta$ in the second exponent in the integrand by writing it as

$$
\frac{1}{\langle\eta\rangle}\left(\eta+i c_{s} \frac{p l_{s}}{\hbar}\left\langle n_{s}\right\rangle\right)^{2}+c_{s}^{2}\left(\frac{p l_{s}}{\hbar}\right)^{2}\left\langle n_{s}\right\rangle,
$$

and then displacing the variable of integration, $\eta^{\prime} \equiv \eta$ $+i c_{s} p l_{s}\left\langle n_{s}\right\rangle / \hbar$, both the integrals adopt the standard form of the Gaussian integral. Accordingly, the above integral yields:

$$
e^{-c_{s}^{2}\left(p l_{s} / \hbar\right)^{2}\left\langle n_{s}\right\rangle}
$$

a) On leave from the Saha Institute of Nuclear Physics, Calcutta, India. 'C. Kittel, Quantum Theory of Solids (Wiley, New York, 1966), pp. 386-388.

${ }^{2}$ W. E. Lamb, "Capture of Neutrons by Atoms in a Crystal," Phys. Rev. 55, 190-197 (1939).

${ }^{3}$ R. L. Mössbauer, "Kernresonanzfloureszenz,” Z. Phys. 151, 124-144 (1958); "Kernresonanzabsorption," Z. Naturforsch. 14a, 211-216 (1959).

${ }^{4}$ V. M. Visscher, "Study of Lattice Vibrations by Resonance Absorption of Nuclear Gamma Rays," Ann. Phys. 9, 194-210 (1960).

'H. J. Lipkin, "Some Simple Features of the Mössbauer Effect," Ann. Phys. 9, 332-339 (1960).

6V. F. Weisskopf, "Selected Topics in Theoretical Physics," in Lectures in Theoretical Physics, edited by W. E. Britten, D. W. Downs, and J. Downs (Interscience, New York, 1960), Vol. III, pp. 70-80.

${ }^{7}$ K. S. Singwi and A. Sjölander, "Resonance Absorption of Nuclear Gamma rays and the Dynamics of Atomic Motions," Phys. Rev. 120, 1093-1102 (1960).

${ }^{8}$ L. van Hove, "Correlations in Space and Time and Scattering from a
System of Interacting Particles," Phys. Rev. 95, 249-262 (1954).

${ }^{9} \mathrm{H}$. Frauenfelder, The Mössbauer Effect (Benjamin, New York, 1963), pp. 26-30.

${ }^{10} \mathrm{~B}$. Kaufman and H. J. Lipkin, "Momentum Transfer to Atoms in a Crystal," Ann. Phys. 18, 294-309 (1962).

"S. Howard and S. K. Roy, "Coherent States of a Harmonic Oscillator," Am. J. Phys. 55, 1109-1117 (1987).

${ }^{12}$ E. Schrödinger, "From Micro- to Macro-mechanics," Naturwiss. 14, 664-676 (1926).

${ }^{13}$ J. R. Klauder and E. C. G. Sudarshan, Fundamentals of Quantum Optics (Benjamin, New York, 1968), pp. 135-146.

${ }^{14}$ R. J. Glauber, Quantum Optics (Academic, New York, 1969), pp. $15-56$.

${ }^{15}$ J. Perina, Coherence of Light (Van Nostrand, New York, 1971), pp. 160-187.

${ }^{16} \mathrm{~J}$. S. Langer, "Coherent States and Superfluidity," Phys. Rev. 184, 219-229 (1969).

${ }^{17}$ J. C. Botke, D. J. Scalapino, and R. L. Sugar, "Coherent States and Particle Production," Phys. Rev. D 9, 813-823 (1974).

${ }^{18}$ G. F. Filippov, V. S. Vasilevski, and L. L. Chopovskii, "Generalized Coherent States in Nuclear Physics," Sov. J. Part. Nucl. 15(6), 600619 (1984).

${ }^{19}$ S. Baba Pundari, B. Dutta-Roy, and G. Ghosh, "The Coherent State and Radial Admixtures in the Ground State Wavefunction of ${ }^{4} \mathrm{He}, " \mathrm{~J}$. Phys. D 9, 813-817 (1976).

${ }^{20}$ R. Gilmore and D. H. Feng, "Coherent States for Bosons and Fermions: A Tutorial," in Progress in Particle and Nuclear Physics, edited by D. Wilkinson (Pergamon, Oxford, 1983), Vol. 9, pp. 479-509.

${ }^{21}$ N. Papanicolaou, "Infra-red Problems in Quantum Electrodynamics," Phys. Rep. 24C, 229-313 (1976).

${ }^{22} \mathrm{P}$. H. Handel, in Noise in Physical Systems and $1 / f$ Noise, edited by A. D'Amico and P. Mazzetti (North Holland, Amsterdam, 1986), pp. $465-469$.

${ }^{23}$ G. Ghosh, B. Dutta-Roy, and D. Bhaumik, "The Coherent State Approach to the Vlasov Equation," Plasma Phys. 19, 1051-1056 (1977).

${ }^{24} \mathrm{~K}$. Bhaumik and B. Dutta-Roy, "The Classical Non-Linear Oscillator and the Coherent State," J. Math. Phys. 16, 1131-1134 (1975).

${ }^{25} \mathrm{G}$. Ghosh and B. Dutta-Roy, "The Berry Phase and the Hannay Angle," Phys. Rev. D 37, 1709-1712 (1988).

${ }^{26}$ J. R. Klauder and B-S Skagerstam, Coherent States (World Scientific, Singapore, 1985).

${ }^{27}$ C. Cohen-Tannoudji, B. Diu, and F. Laloë, Quantum Mechanics, Vol. I (Wiley, New York, 1977), pp. 295-307.

\section{SHAKESPEARE AND THE SECOND LAW OF THERMODYNAMICS}

A good many times I have been present at gatherings of people who, by the standards of the traditional culture, are thought highly educated and who have with considerable gusto been expressing their incredulity at the illiteracy of scientists. Once or twice I have been provoked and have asked the company how many of them could describe the Second Law of Thermodynamics. The response was cold: it was also negative. Yet I was asking something which is about the scientific equivalent of: Have you read a work of Shakespeare's?

C. P. Snow, The Two Cultures and the Scientific Revolution (Cambridge, U. P., New York, 1959), pp. 15-16. 